\title{
Molecularly Imprinted Microspheres for the Remediation of 2,4- Dichlorophenol from Wastewater Using 'Template Analogue Imprinting Strategy'
}

\author{
Santwana Dhongade ${ }^{\mathrm{a}}$, Sushma Pardeshi ${ }^{\mathrm{a}}$, Anupama Kumar ${ }^{\mathrm{a}}$ * \\ ${ }^{a}$ Chemistry Department, Visvesvaraya National Institute of Technology, Nagpur, 440010, India
}

\begin{abstract}
Molecularly imprinted polymers (MIPs) with high selectivity and binding capacity for the template Gallic acid (GA) was used as an adsorbent for the binding of 2, 4-dichlorophenol (2, 4-DCP) for wastewater remediation. Computational modeling was used to probe the nature of interaction between the functional monomer acrylamide and the template analogue 2, 4-DCP. The morphological characterization of MIPs synthesized by the reverse microemulsion polymerization was done using Scanning electron microscopy (SEM), BET surface area characterization and Fourier transform infrared spectroscopy (FTIR). The binding capacities of MIP and NIP for the template GA were $883 \mu_{\mathrm{molg}}{ }^{-1}$ and $296 \mu \mathrm{molg}^{-1}$ respectively indicating the successful imprinting of GA. However, for 2, 4-DCP, the binding capacities of MIP and NIP were $275 \mu$ molg $^{-1}$ and 268 umolg ${ }^{-1}$ respectively which indicated the unspecific adsorption of 2, 4-DCP by the polymers. MIP \& NIP although exhibited unspecific binding for 2, 4-DCP however they were found to be highly efficient for depletion of this hazardous compound which has low permissible limits from real wastewater samples.
\end{abstract}

Keywords: Template analogue imprinting strategy;Molecularly imprinted polymer, 2, 4-dichlorophenol, Reverse microemulsion polymerization; Microspheres

\section{Introduction}

The Molecularly imprinted polymers (MIPs) are the macromolecular materials with the tailor-made binding sites for the template molecule. MIPs are used in wide range of applications as it offers easy and economical synthesis, robust mechanical properties and selectivity for the target analyte [1,2].Template analogue imprinting strategy also known as Dummy template imprinting strategy is the use of structurally similar compound of the target analyte as the template for imprinting [3,4]. This strategy is efficient, economic and safe for templates which are either costly or hazardous (pollutants). The objective of the present study is to investigate the potential of Gallic acid (GA) imprinted polymers to bind the 2, 4-dichlorophenol (2, 4-DCP) from the aqueous matrix envisaging the multi usage of the GA imprinted polymers. The morphology, binding capacity and regeneration ability of MIPs were also investigated.

2, 4-DCP is one of the important chlorophenols since, it is used extensively in the manufacturing of pesticides and herbicides [5]. It is considered as an environmental pollutant which causes serious ecological problems due to its high toxicity, strong odor emission and persistence in the environment. It is reported to have suspected carcinogenic and mutagenic effects on the living organisms. It is considered as priority pollutants by the US EPA in the Clean Water Act $[6,7,8]$. The source of 2, 4-DCP in the environment is the wastewater coming from petroleum refineries, plastics, rubbers, pharmaceuticals and steel industries [9,10]. Discharge of 2 , 4-DCP contaminated wastewater into the environment without adequate treatment can lead to negative effect on the water quality. The permissible limit for the 2, 4-DCP in water sources has been reported as $0.25 \mu \mathrm{g} / \mathrm{L}$ for fresh water and $1 \mu \mathrm{g} / \mathrm{L}$ for drinking water [11]. Several methods including chemical oxidation [12], ion exchange [13], adsorption [10] and biological degradation [10] has been proposed for the removal of 2, 4-DCP. These methods have high treatment costs and have low efficiency for removing 2, 4-DCP. Thus, a low-cost and highly effective removal or depletion methods is essential for removal of 2, 4-DCP from the water matrix. Due to desired predetermination by theoretical and computational studies [14], favorable thermal, mechanical and chemical stability along with simple preparation technique and economic efficiency, MIPs finds applications in wide range of fields such as separation, chemical sensing, solid phase extraction and many other applications $[15,16]$. In the present work we intend to use the GA based MIPs for removal of 2, 4-DCP on the basis of template analogue imprinting strategy.

\subsection{Reagents and analytical instruments}

\section{Materials and methods}

GA, 2, 4-DCP, Span-80 (sodium monooleate) and magnesium sulfate were purchased from SigmaAldrich (Buchs, Switzerland). Ethylene glycol dimethacrylate (EGDMA), Acrylamide were purchased from Merck (Darmstadt, Germany) and 2, 2-azoisobutyronitrile (AIBN) from National Chemicals (India). AIBN was 
re-crystallized using methanol before use. All the solvents were reagent grade or HPLC-grade and purchased from Merck (India) and used without further purification. Double distilled, deionized water was used for experiments. Analysis was performed using UV-Visible spectrophotometer (UV-1800, Shimadzu, Japan) with matched $1 \mathrm{~cm}$ quartz cells. The calibration curve of 2, 4-DCP was prepared in the water at $284 \mathrm{~nm}$ for binding experiments. Waters 2535 Quaternary Gradient Module with Waters 2998 Photodiode array detector was used for HPLC analysis.

\subsection{Preparation of template analogue imprinted and non-imprinted polymers}

$0.5 \mathrm{~mL}$ Span 80 emulsifier was dissolved in $20 \mathrm{~mL}$ acetonitrile (porogen) in a $250 \mathrm{~mL}$ three necked polymerization flask before adding $40 \mathrm{mmolL}^{-1}$ of cross-linker EGDMA, for preparation of template analogue MIP. $1 \mathrm{mmolL}^{-1} \mathrm{GA}$ and $4 \mathrm{mmolL}^{-1}$ of acrylamide was dissolved in $10 \mathrm{~mL}$ distilled water and added to the acetonitrile solution. The mixture was sonicated for 5 min to provide water-in-oil emulsions. Then, $150 \mathrm{~mL}$ aqueous solution of magnesium sulfate $\left(0.015 \mathrm{molL}^{-1}\right)$ was added followed by addition of $7 \mathrm{mmolL}^{-1} \mathrm{Na}$-DBS. The mixture was sonicated for $5 \mathrm{~min}$ to obtain water-in-oil-in-water emulsions. After the addition of $0.5 \mathrm{~g}$ AIBN as the initiator, polymerization was carried out in a rota-mantle at $70{ }^{\circ} \mathrm{C}$ for 6 hours under nitrogen protection with mild stirring. The resultant polymer product was collected by centrifugation at $4500 \mathrm{rpm}$ for 10 minutes and dried at $70{ }^{\circ} \mathrm{C}$.

The non-imprinted polymer (NIP) was also prepared by using the same synthetic protocol except addition of GA. MIP and NIP were washed with methanol-acetic $(50: 50 \mathrm{v} / \mathrm{v})$ once, followed by several washings with distilled water to remove stabilizer and emulsifier as well as unreacted precursors. MIP was washed using methanol: acetic acid $(80: 20 \mathrm{v} / \mathrm{v})$ in a soxhlet extractor to remove GA from the polymeric matrix. The washing cycles were repeated till no desorption of GA was detected in the eluent. Further, MIP was washed with solution of $0.1 \mathrm{mmolL}^{-1} \mathrm{Na}_{2} \mathrm{CO}_{3}$ followed by distilled water to remove the residual acetic acid. Finally, MIP and NIP were dried at $60{ }^{\circ} \mathrm{C}$ and stored at ambient temperature for further experiments and characterization.

\subsection{Quantum chemical computational analysis}

\section{Analysis}

The ground state molecular structure of 2, 4-DCP, Acrylamide and their subsequent complexes were optimized using the B3LYP/ 6-31G(d,p) basis set using Gaussian 09 program package [17]. Geometry optimizations were followed by frequency calculations to confirm the structures as minima with no negative frequencies and to obtain the Gibbs free energies. The change in the Gibbs free energy $(\Delta \mathrm{G})$ due to the complex formation was calculated using Equation (1).

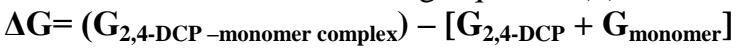

Where, $\Delta G$ is the change in Gibbs free energy on the formation of 2,4-DCP - monomer complex, $\left(\mathrm{G}_{2,4-\mathrm{DCP}}\right.$ monomer complex $)$ is the Gibbs free energy of 2,4-DCP - monomer complex, $\left(\mathrm{G}_{2,4-\mathrm{DCP}}\right)$ is the Gibbs free energy of 2,4-DCP and $\left(\mathrm{G}_{\mathrm{monomer}}\right)$ is the Gibbs free energy of functional monomer.

\section{Results}

4.1. DFT based Quantum chemical computational modeling

The binding mechanism of 2, 4-DCP to polymers was investigated using computational modeling. The thermodynamic properties of 2, 4-DCP and Acrylamide and their complex are presented in TABLE 1.

Table 1 - Thermodynamic parameters of 2, 4- DCP, Acrylamide and their complex

\begin{tabular}{|c|c|c|c|}
\hline Thermodynamic parameters & 2, 4- DCP & Acrylamide & $\begin{array}{l}\text { 2, 4- DCP- Acrylamide } \\
\text { complex }\end{array}$ \\
\hline Electronic energy including zero-point correction (E a.u.) & -1226.6 & -247.3 & -1473.8 \\
\hline Gibb's free energy (G a.u.) & -1226.6 & -247.3 & -1473.9 \\
\hline Total energy (thermal) $\left(\mathrm{kcalmol}^{-1}\right)$ & 58.8 & 52.6 & 113.9 \\
\hline Heat capacity $(\mathrm{CV})(\mathrm{cal} / \mathrm{mol} \mathrm{K})$ & 29.4 & 17.3 & 52.8 \\
\hline Entropy (S) (cal/mol K) & 88.5 & 71.6 & 130.3 \\
\hline Translational energy $\left(\mathrm{kcalmol}^{-1}\right)$ & 0.89 & 0.89 & 0.89 \\
\hline Rotational energy $\left(\mathrm{kcalmol}^{-1}\right)$ & 0.89 & 0.89 & 0.89 \\
\hline Vibrational energy $\left(\mathrm{kcalmol}^{-1}\right)$ & 56.9 & 50.9 & 112.2 \\
\hline \multicolumn{4}{|l|}{ Dipole moment ( $\mu$ Debye) } \\
\hline$\mu x$ & 0.96 & 0.27 & -4.60 \\
\hline$\mu y$ & 0.46 & -3.51 & -3.05 \\
\hline$\mu \mathrm{z}$ & 0.0001 & 0.0438 & -1.2211 \\
\hline$\mu$ total & 1.07 & 3.53 & 5.65 \\
\hline
\end{tabular}




\subsection{Morphological characterization of polymers}

The samples were analyzed using a Scanning electron microscope (JEOL JSM-6360LV). The samples were coated with a thin gold film before observation. SEM micrographs of the MIPs and NIPs are presented in Fig. 1 and Fig. 2 respectively.

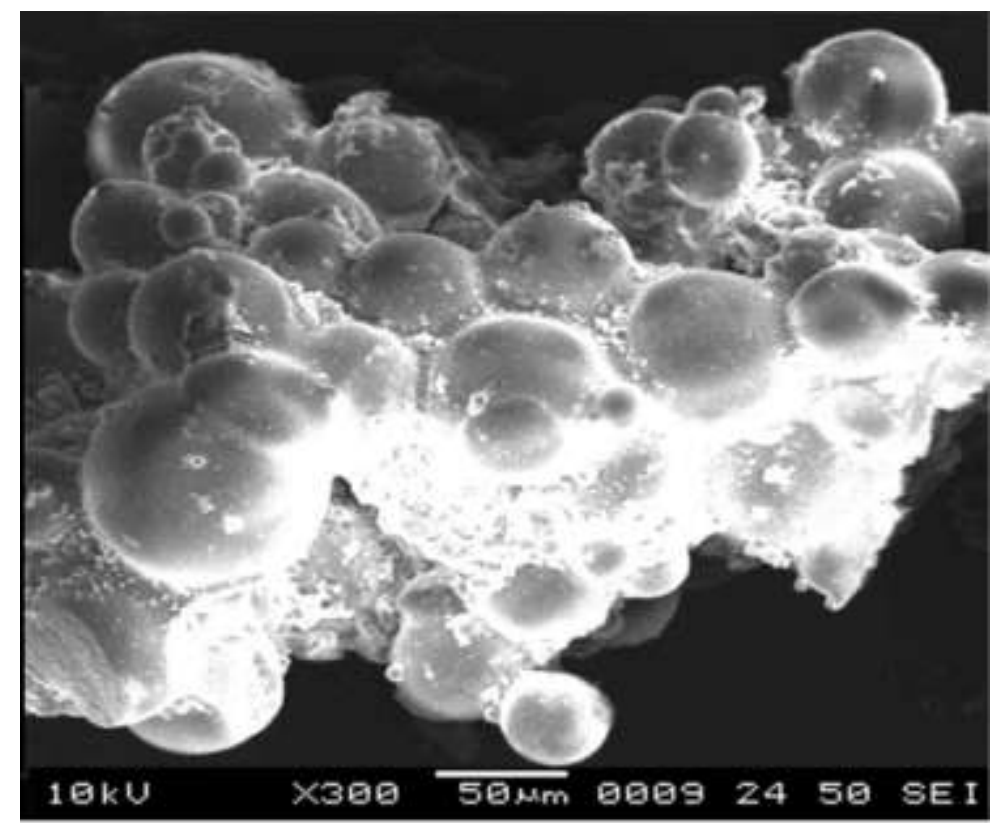

Figure 1: SEM micrograph for MIPs

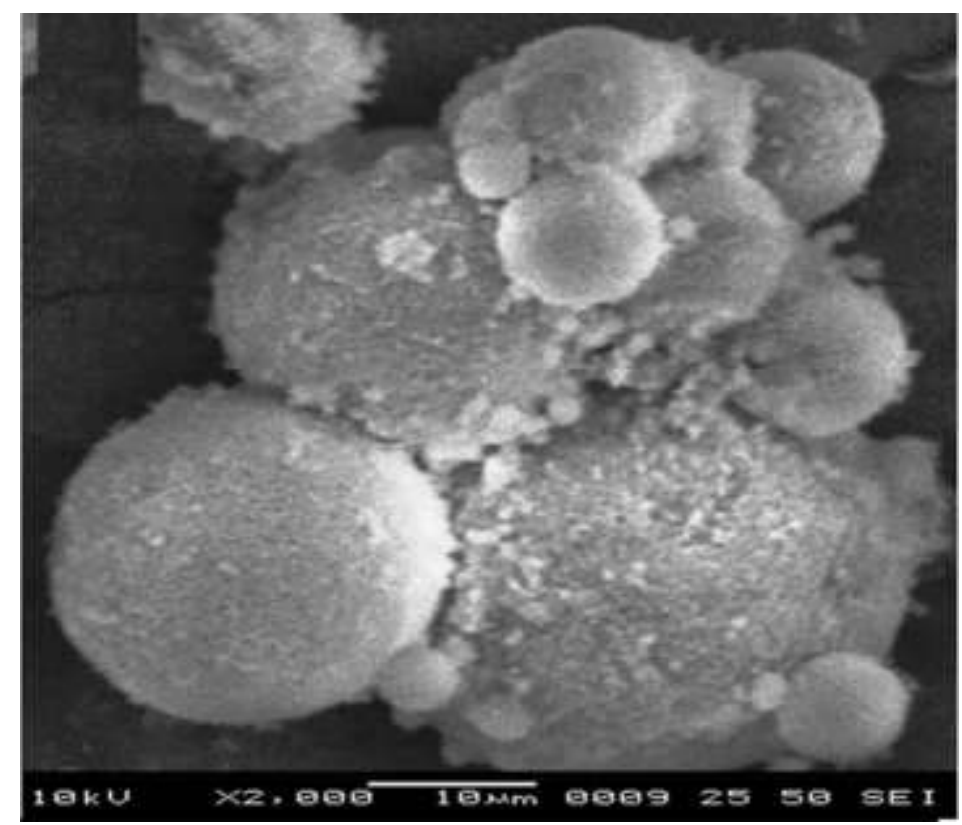

Figure 2: SEM micrograph for NIPs

Surface area analysis was performed on Surface area analyzer: Smart Sorb 92/93. The samples were degassed for 4 hours at $100{ }^{\circ} \mathrm{C}$ before analysis. The surface area of the MIP and NIP were found to be 254.21 $\mathrm{m}^{2} \mathrm{~g}^{-1}$ and $138.18 \mathrm{~m}^{2} \mathrm{~g}^{-1}$, respectively. FT-IR spectra of compounds were determined using a Fourier Transform Infrared Spectrometer (IR-Affinity-1, Shimadzu). A small quantity of sample was ground with anhydrous potassium bromide and the spectra were recorded between 4000 and $400 \mathrm{~cm}^{-1}$, by averaging 45 scans for each spectrum. The FTIR spectra of MIP after removal of GA (Fig. 3 a), NIP (Fig. 3 b) and 2, 4-DCP bound MIP (Fig. 3 c) were obtained. 


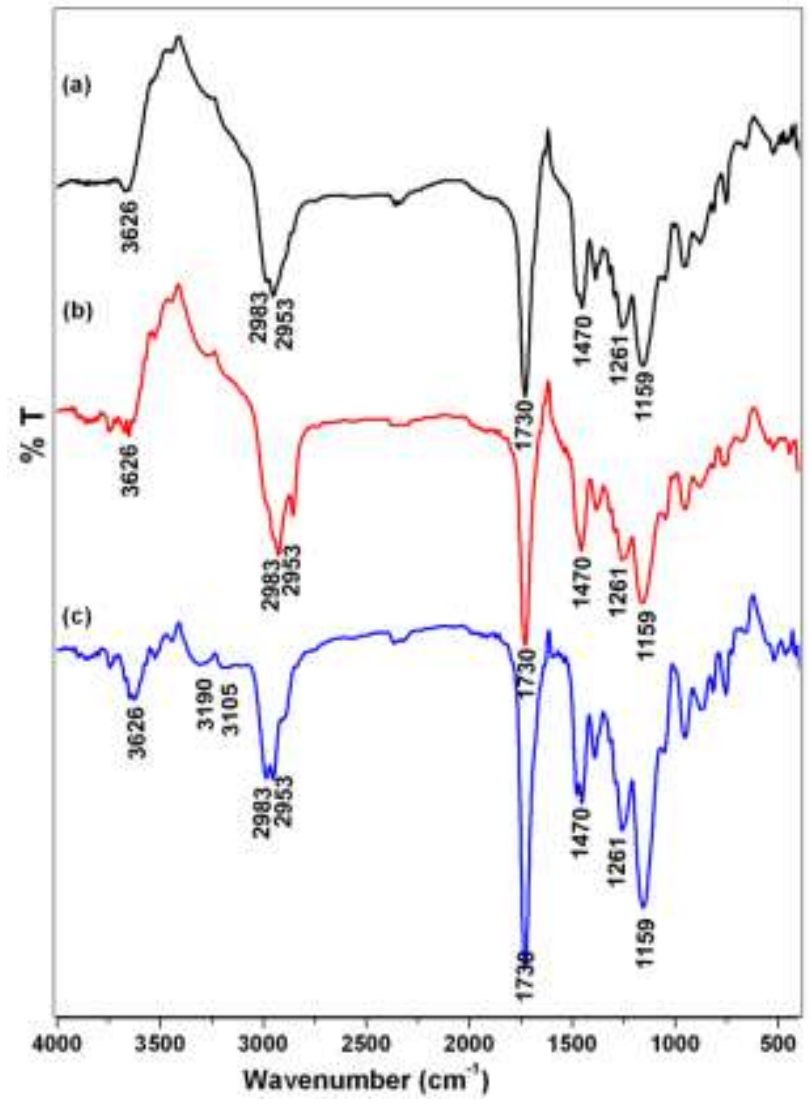

Figure 3: FTIR spectra (a) MIP after removal of GA, (b) NIP, (c) 2, 4-DCP bound MIP

\subsection{Binding study}

\subsubsection{Effect of $\mathrm{pH}$ on the binding capacity of MIP}

Optimization of $\mathrm{pH}$ is important when the binding is to be performed in the aqueous medium. The binding capacity of MIP was determined at different $\mathrm{pH}$ as presented in Fig. 4.

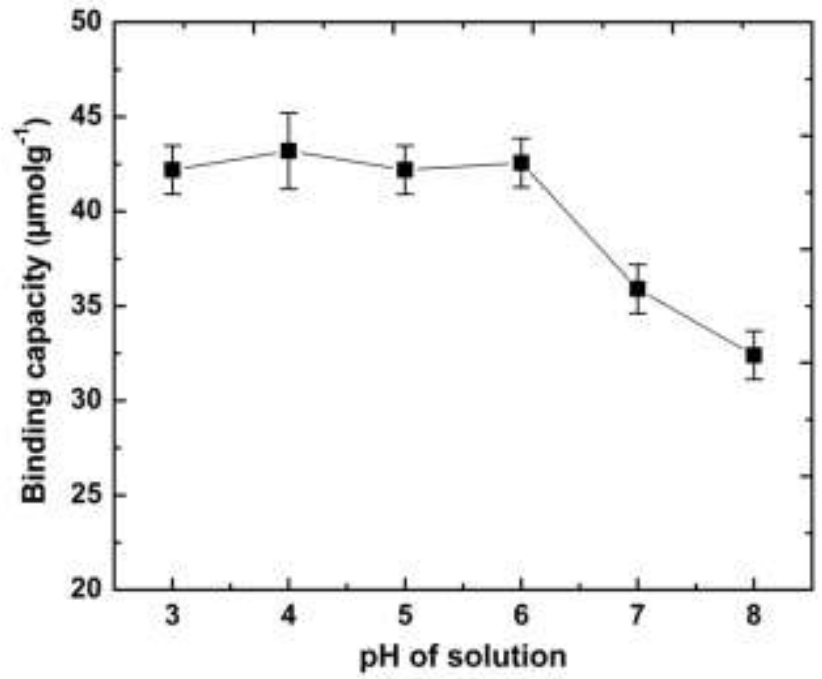

Figure 4: Effect of $\mathrm{pH}$ on binding capacity of MIPs

\subsubsection{Effect of time on the binding capacity of MIP}

Time is the most significant factor for any experiment. Hence it is necessary to optimize time required for highest binding capacity of polymer. The effect of varying time on the binding capacity of polymers is shown in Fig. 5. 


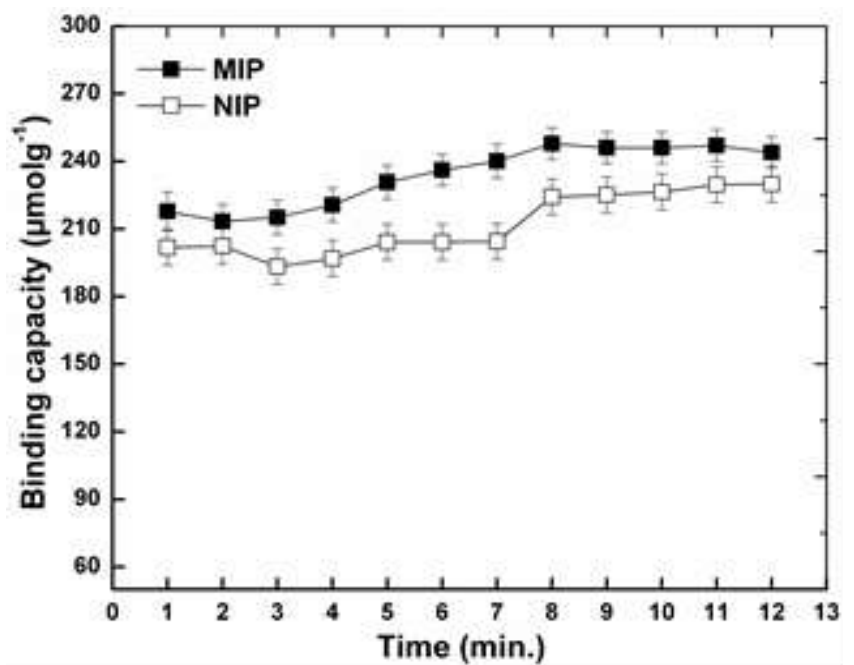

Figure 5: Effect of time on binding capacity of MIPs and NIPs

\subsubsection{Effect of concentration on the binding capacity of MIP}

It is necessary to find out the interaction of polymer with increasing concentration of targeted analyte. This helps to determine the highest possible concentration for extraction or depletion of the target template by the polymer. The binding capacity of MIP and NIP was determined at varying concentrations of 2, 4-DCP as presented in Fig. 6 and Fig. 7 respectively.

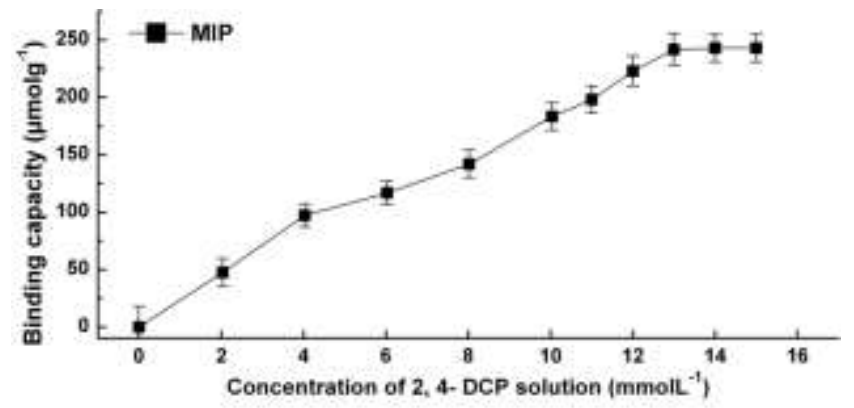

Figure 6: Effect of concentration on binding capacity of MIPs

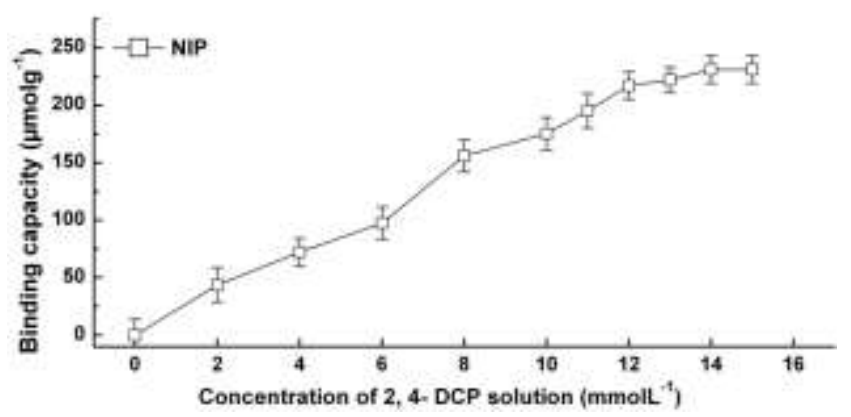

Figure 7: Effect of concentration on binding capacity of NIPs

\subsubsection{Application of polymers to real sample of wastewater for depletion of 2, 4-DCP}

Standard solution of 2, 4-DCP $\left(10 \mathrm{mgL}^{-1}\right)$ was injected into HPLC system and the retention time was found to be $6.5 \mathrm{~min}$ (flow rate-1 $\mathrm{mL} / \mathrm{min}$ and injection volume $10 \mu \mathrm{L}$ ). Wastewater sample was collected and filtered several times to remove all the particulate impurities and injected into HPLC system. The peak at 6.5 min. in the chromatogram of wastewater was well matched with the peak of standard 2, 4-DCP. The concentration of 2, 4-DCP in the wastewater sample was found to be $16 \mathrm{mgL}^{-1}$. The $\%$ RSD for intermediate precision (inter-day precision) and repeatability (intra-day precision) was found to be $1.49 \%$ and $1.39 \%$ respectively. Accuracy of the method was assessed by spiking the wastewater sample with the 2, 4-DCP. Recovery values were obtained between $98 \%$ and $101 \%$, which indicated a satisfying accuracy of the method. 


\subsubsection{Regeneration ability of polymers}

The regeneration of adsorbent is a key factor for economy and reuse. The binding capacity of MIP decreased only up to $20 \%$ after five sequential cycles of regeneration and reuse. The results indicated that the polymers could be used repeatedly for depletion of 2, 4-DCP from the wastewater sample.

\section{Discussion}

\subsection{DFT based quantum chemical computational modeling}

The acrylic acid and acrylamide exhibited high interaction energy with the GA as reported in the previous study [18]. The nature of interaction of 2, 4-DCP with the monomers acrylic acid and acrylamide was investigated using DFT based quantum chemical computational modeling. The change in the Gibbs free energy $(\Delta \mathrm{G})$ as a measure of binding between 2, 4-DCP and acrylic acid was found to be $-8.53 \mathrm{kcalmol}^{-1}$. The $\Delta \mathrm{G}$ between 2, 4-DCP and acrylamide was found to be $-12.29 \mathrm{kcalmol}^{-1}$. Thus it was observed that the acrylamide exhibited better interaction with 2, 4-DCP as compared to the acrylic acid.

2, 4-DCP and monomer acrylamide are polar in nature as evident from the values of dipole moment (Table 1). The dipole moment of the 2, 4-DCP was in the direction of $\mathrm{Cl}$ group which is attached at fourth position of benzene ring. It pulled the electrostatic field surrounding the $-\mathrm{OH}$ group thus formation of the hydrogen bonds or dipole-dipole interactions through this $-\mathrm{OH}$ group is possible.

\subsection{Morphological characterization of polymers}

It was observed that the MIPs and NIPs were formed as microspheres of size 15 to $20 \mu \mathrm{m}$. The morphology of polymer beads aroused from the nuclei formed around the initiator during the polymerization process, which grew to $10-30 \mathrm{~nm}$ in diameter and then formed the microspheres. Further, these microspheres might aggregate themselves into larger clusters that form the body of beads. The microspheres provided higher surface area resulting into better binding efficiency of 2, 4-DCP.

The N-H stretching of amide group due to incorporation of monomer acrylamide was observed at 3626 $\mathrm{cm}^{-1}$ in MIP and NIP (Figure $3 \mathrm{a}$ and b). The major peaks observed in the polymers were : C-H stretching at $2983 \mathrm{~cm}^{-1}$ and $2953 \mathrm{~cm}^{-1}, \mathrm{C}=\mathrm{O}$ stretching at $1730 \mathrm{~cm}^{-1}, \mathrm{C}-\mathrm{H}$ bending at $1470 \mathrm{~cm}^{-1}$, C-C stretching at $1261 \mathrm{~cm}^{-1}$ and $\mathrm{C}-\mathrm{O}$ stretching at $1159 \mathrm{~cm}^{-1}$. These peaks were mainly due to the incorporation of cross-linker EGDMA in the polymer matrix. The IR spectra of MIP showed close resemblance to NIP after the removal of the GA from the MIP matrix. The spectra of MIP after binding 2, 4-DCP (Figure $3 \mathrm{c}$ ) showed no appreciable difference in the morphology except appearance of small bands in the region $3190 \mathrm{~cm}^{-1}$ to $3105 \mathrm{~cm}^{-1}$ which corresponds to the aromatic C-H stretching of 2, 4-DCP bound to the polymer. FTIR data depicted the successful synthesis of the MIP and NIP.

\subsection{Binding study}

\subsubsection{Effect of $\mathrm{pH}$ on binding capacity of MIP}

In this experiment, it was observed that binding capacity remained constant in the $\mathrm{pH}$ range of 3 to 6 followed by a gradual decrease at higher $\mathrm{pH}$. Thus, the further studies were performed at $\mathrm{pH} 6$.

\subsubsection{Effect of time on binding capacity of MIP}

From the Figure 5, it can be observed that almost $90 \%$ binding was achieved in the first minute of equilibration and it increased slightly up to $8 \mathrm{~min}$. Thereafter, it became steady for the further time duration. 12 minutes was chosen as the optimized time to ensure the maximum binding. The other important observation was that the NIP exhibited almost equal binding capacity to the MIP. This trend demonstrated that the binding of 2, 4-DCP by the polymers was purely unspecific in nature. This may be due to the imprinting pockets within the MIP which were more selective for the template analogue GA as compared to 2, 4-DCP.

Thus, both the MIP and NIP were able to bind the 2, 4-DCP.

\subsubsection{Effect of concentration on binding capacity of MIP}

Figure 6 and 7 indicated that the binding capacity of polymers (MIP and NIP) increased with the increase in the concentration and reached maximum at concentration of $14 \mathrm{mmolL}^{-1}$. Thereafter, it remained steady. The binding capacity of MIP was $243 \mu \mathrm{molg}^{-1}$ and the binding capacity of NIP was $231 \mu \mathrm{molg}^{-1}$ at the concentration of $14 \mathrm{mmolL}^{-1}$. The binding capacity of MIP and NIP for the original template GA under the identical conditions were $883 \mu \mathrm{molg}^{-1}$ and $296 \mu \mathrm{molg}^{-1}$ respectively resulting into the specific binding capacity (binding capacity of MIP-binding capacity of NIP) of $587 \mu \mathrm{molg}^{-1}$ due to the imprinting of GA. The observed trend of 2, 4- DCP binding by the polymers is advantageous as both MIP and NIP can be utilized for the binding of 2, 4-DCP. 


\subsubsection{Application of polymers to real sample of waste water for depletion of 2, 4-DCP}

According to the data of binding experiments, a protocol for the binding of 2, 4-DCP from the real matrix of wastewater was developed. The wastewater sample was spiked with 2, 4-DCP in order to reach a final concentration of $14 \mathrm{mmolL}^{-1}$, in line with previous findings (Figure 5).25 mg of the MIP \& NIP were equilibrated separately with the spiked wastewater sample for 12 minutes. The samples were centrifuged, filtered and injected into the HPLC system. The percent binding capacity of MIP \& NIP was found to be $82.3 \%$ and $74.8 \%$, respectively from the wastewater sample. The maximum permissible limit of 2, 4-DCP in drinking water is $1 \mu \mathrm{g} / \mathrm{L}$ [11]. The template analogue MIP and NIP were found to be efficient for removal of 2, 4-DCP and can prove useful in depletion of hazardous 2, 4-DCP from environmental samples.

\section{Conclusion}

In the present investigation, uniformly sized molecularly imprinted microspheres (MIP) were successfully prepared by the reverse micro-emulsion polymerization method using GA as template analogue. The MIP was evaluated as adsorbent for removal of 2, 4-DCP from aqueous solutions. The template analogue MIP and NIP were found to highly efficient for the depletion of 2, 4-DCP from the wastewater matrix. This study indicated multi-usage of the template analogue MIPs and NIPs for remediation of wastewater.

\section{Acknowledgements}

The authors acknowledge Department of chemistry, VNIT, Nagpur for providing uninterrupted laboratory facilities. We are highly thankful to Ms. G. Archana for her support during sampling, sample preparation and High Performance Liquid Chromatography analysis.

\section{References}

[1]. S. Pardeshi, R. Dhodapkar, A. Kumar, Quantum chemical density functional theory studies on the molecular structure and vibrational spectra of Gallic acid imprinted polymers Spectro. Chim. Acta. Mol. Biomol. 116, 2013, 562-573.

[2]. Z. Lin, W. Cheng, Y. Li, Z. Liu, X. Chen, C. Haung, A novel superparamagnetic surface molecularly imprinted nanoparticle adopting dummy template: An efficient solid-phase extraction adsorbent for bisphenol A, Anal. Chim. Acta. 720, 2012, 71-76.

[3]. H.T. Zhang, T. Song, W. Zhang, W. Hua, C. P. Pan, Retention behavior of phenoxyacetic herbicides on a molecularly imprinted polymer with phenoxyacetic acid as a dummy template molecule Bioorg. Med. Chem. 15, 2007, 6089-6095.

[4]. J. Li, X. Zhang, Y. Liu, H. Tong, Y. Xu, S. Liu, Preparation of hollow porous molecularly imprinted polymer using tetrabromobisphenol A as a dummy template and its application as SPE sorbent for determination of Bisphenol A in tapwater, Talanta, 117, 2013, 281-287.

[5]. J. Pan, L. Xu, J. Dai, X. Li, H. Hang, P. Huo, C. Li, Y. Yan, Magnetic molecularly imprinted polymers based on attapulgite/Fe3O4 particles for the selective recognition of 2,4-dichloropheno Chem. Eng. J. 174, 2011, 68-75.

[6]. J. Zhang, J. Lei, H. Ju, C. Wang, Electrochemical sensor based on chlorohemin modified molecularly imprinted microgel for determination of 2,4-dichlorophenol Anal. Chim. Acta., 2013, 786, 16-21.

[7]. X. Lv, Yonghao Wang, Yongjing Wang, Z. Lin, Study of Photocatalytic Degradation of 2, 4-Dichlorophenol by ZnS Microsphere J. Nanosci. and Nanotech. 16, 2016, 1060-1066.

[8]. L. O. Conte, A. V. Schenone, O. M. Alfano, Photo-fenton degradation of herbicide 2, 4-D in aqueous medium at pH conditions close to neutrality J. Environ. Manage. 170, 2016, 60-69.

[9]. J. F. Peng, J. F. Liu, X. L. Hu, G.B. Jiang, Direct determination of Chlorophenols in Environmental water samples by hollow fibre supported ionic liquid membrane extraction coupled with high performance liquid chromatography J. Chromato. A. 1139, 2007, $165-170$

[10]. F. Ren, R. Zhang, W. Lu, T. Zhou, R. Han, S. Zhang, Adsorption potential of 2, 4-Dichlorophenol onto cationic surfactant modified phoenix tree leaf in batch mode Desali. and Water Treat. 57, 2016, 6333-6346.

[11]. K. S. Lin, H. P. Wang, M. C. Li, Oxidation of 2, 4-dichlorophenol in supercritical water, Chemosphere, 36, $1998,2079-2083$.

[12]. X. Cheng, H. Guo, Y. Zhang, Y. Liu, H. Liu, Y. Yang, Oxidation of 2,4-Dichlorophenol by non-radical mechanism using persulfate activated by Fe/S modified carbon nanotubes J. Colloids and Inter. Sci., 469, 2016, 277-286.

[13]. M. Akhtar, M. I. Bhanger, S. Iqbal and S. M. Hasany, Sorption potential of rice husk for the removal of 2, 4-dichlorophenol from aqueous solutions: kinetic and thermodynamic investigations, J. Hazard. Mater. 128, 2006, 44-52.

[14]. X. Wei, Z. Zhou, T. Hao, H. Li, Y. Xu, K. Lu, Y. Wu, J. Dai, J. Pan, Y. Yan, Highly-controllable imprinted polymer nanoshell at the surface of silica nanoparticles based room-temperature phosphorescence probe for detection of 2,4-dichlorophenol Anal. Chim. Acta. 870, 2015, 83-91.

[15]. P. Yu, Q. Sun, J. Li, Z. Tan, Y. Yan, C. Li, Selective removal of 2,4-dichlorophenol in aqueous solutions by nanoparticles modified yeast during miniemulsion imprinting polymerization, J. Env. Chem. Eng. 3, 2015, 797-806.

[16]. V. B. Kandimalla, H. Ju, Molecular imprinting: a dynamic technique for diverse applications in analytical chemistry, Anal. Bioanal. Chem. 380, 2004, 587-605.

[17]. M.J. Frisch et al (2015) Gaussian 09, Revision E.01, Gaussian Inc., Pittsburgh, PA.

[18]. S. Pardeshi, R. Patrikar, R. Dhodapkar, Validation of computational approach to study monomer selectivity toward the template Gallic acid for rational molecularly imprinted polymer design A. Kumar, J. Mol. Model. 18, 2012, 4797-4810. 